\title{
An Online Monitoring System for Frequency Conversion Speed Regulating Fans Applied in the Coal Mine
}

\author{
Xiaoxi Zheng ${ }^{1, a}$, Hui Wang ${ }^{1, b}$, Shuaifei Shao ${ }^{2, c}$, Mengcai Guo ${ }^{3, d}$ \\ 1 Zhengzhou Technical College,Zhengzhou 450021,China; \\ 2 Sippr engineering group CO.LTD, Zhengzhou 450001, China; \\ 3Henan east China industrial control technology co., LTD ,Zhengzhou 450001, China \\ a zxx20028@163.com, ${ }^{b} 951980613 @ q q . c o m, ~ ' d a n g d a n g l u @ 163 . c o m,{ }^{d}$ guomc291@163.com
}

Keywords: PLC,Fan,mine safety, e-monitoring system, frequency conversion .

\begin{abstract}
Based on the situation of the ventilation system applied in the coal mine, an online-monitoring system is designed, which focuses on monitoring the parameters of the frequency conversion speed regulating fans. An S7-300 series PLC is employed as the operational core in the proposed monitoring system, which is able to pick up the gas concentration and the key parameters of the fans. The fans are driven by the frequency converter to adjust the rotating speed. All the key operational parameters of the system are transferred and displayed onto the host computer. The functionalities of the proposed system include monitoring the fans' running parameters, operational status and raising failure alarms. The online monitoring system are installed in a coal mine. It shows that the applied system enhances the extent of the automation in the coal mining procedure and gives satisfactory overall performance.
\end{abstract}

\section{Introduction}

According to statistics major accidents of coal mines in our country, mostly due to the unreasonable ventilation system ,which cause methane explosion, coal dust explosion, etc. With the frequent occurrence of coal mine accident, mine production safety is a question that requires great attention. Mine fan for reducing mine work area temperature, supplying fresh air for mine, diluting and removing methanal, dust, is the key equipment to ensure stable and reliable operation of the mine safety production. Fan performance affect the entire mine production and safety because of a closed environment in mine, so the real-time monitoring of mine fan performance and providing information timely to operators, can avoid production accidents and economic losses caused by a malfunctioning fan unit fault, so as to improve the safety of mine production and economy.

\section{System overview of the main fan system}

A mine main fan system uses 2 axial fan with the same function, in which uses one and prepares one. Each fan is driven by Ex Variable-frequency Motor with 8 pole, the motor capacity is $450 \mathrm{~kW}$, the voltage is $10 \mathrm{kV}$. The ventilation system is reversed into the opposite direction through the motor. The fan is also equipped with temperature measuring element Pt100 of motor, Bearing temperature measuring instrument, Desiccant heater of an motor, Damper and device for opening and closing the damper, Ventilation machine parameter(air flow, negative pressure, etc.) test instrument with RS485 interface. The system can realize inverter output between automatic and manual frequency, so that the work of the fan in the power frequency or anging frequency; At the same time, the system can monitor the real-time data of the fan running state and the timely warning and timely processing of the fault. The parameters of fan monitoring are:

(1) The temperature parameters: stator temperature, temperature of the shaft before and after the shaft, the temperature of the fan. 
(2) Electrical parameters: three phase voltage and current, power frequency, active power and reactive power, power factor.

(3) Fan vibration parameters: horizontal and vertical vibration parameters.

(4) The state parameters of the fan: throttle position and motor starting and stopping state parameters.

(5) Fan parameters: fan static pressure, total pressure of the fan, air duct gas and CO concentration, air flow, ambient temperature and humidity and other parameters.

\section{Design of automatic monitoring and control system for main fan}

Fan monitoring system mainly consists of two parts, respectively, automatic adjustment of air flow and automatic monitoring of the running state of the fan. The hardware of the system with high reliability of S7-300 series PLC as the core of the control system, the other part is composed of sensors, transmitters, touch screen, electric parameter acquisition module (including voltage, current transformer), temperature inspection instrument, computer, alarm etc., Through the communication interface connection with fan parameter tester, fan frequency conversion equipment, Intelligent power monitor, gathers the related parameters of damper and fan, upload data to the PC monitoring system and dispatching center of mine, for Continuous monitoring of wind machine, air, gas, pressure and other parameters, The system supports remote programming and remote diagnosis. Using the controller output data signals, converter adjust air volume reasonably according to the data, it ensures the quality of mine air within the prescribed scope, and improve the efficiency of the fan, thus saving resources.

S7 300 series PLC is the core of system which has automatic monitoring and control for the main fan, automatic monitoring and control system is shown in figure 1.

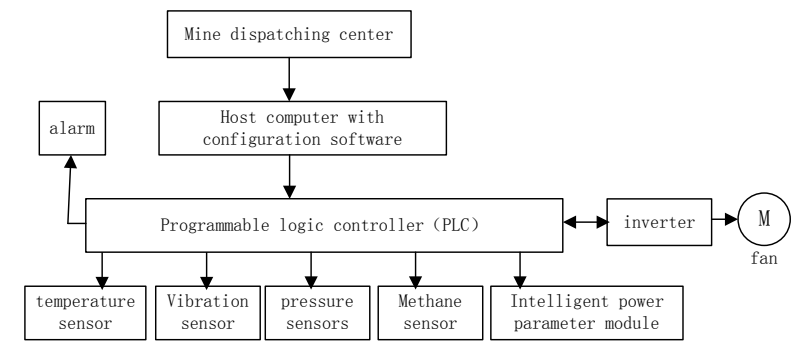

Fig. 1 Main ventilator automatic monitoring system

4. Hardware of the system

\subsection{The selection of PLC module}

This system uses SIEMENS S7 300 series PLC, the hardware includes power module, CPU314 module, 2 digital output module SM322 (DO16 * DC24 V/0.5 A), 4 SM331 analog input module (AI8 * 16 Bit), 3 digital input module SM321 (DI16 * DC24 V). The hardware structure of S7-300 series PLC is shown in Figure 2.

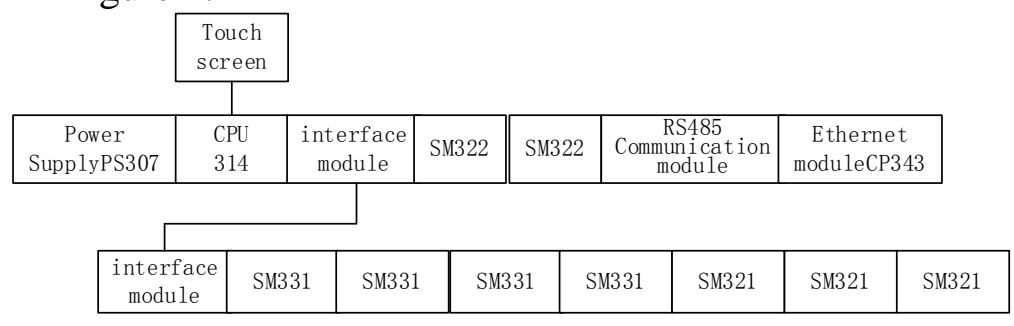

Fig. 2 The hardware structure of S7-300 series PLC

Digital input and output module is used to detect NO 1、NO 2 damper state of contactor, damper opening and closing in place, high and low voltage cabinet control mode, switch state, contactors and motor thermal relay status, running state and fault of inverter, 1, 2 fan of the positive transfer / 
inversion and frequency conversion / frequency start, Control the closing of the switch of high and low pressure tank. The analog input and output module mainly completes the A/D conversion of the converter output speed level current, Fan flow and negative pressure, motor vibration and temperature, High and low voltage power parameters. The analog input, output module, digital input and output modules are all reserved. The communication between the parameter acquisition module and the upper computer is completed by the communication module, The communication module of this system is CP343.

\subsection{Detection of main parameters of the system}

(1) Wind pressure detection

In order to ensure the safe and efficient operation of the wind turbine, the wind pressure and the flow rate of the fans need to be detected. The system pressure detection adopts GPD5 differential pressure sensors and GP5000 negative pressure sensor. Sensor converts pressure or flow into $4 \sim 20 \mathrm{~mA}$ current to the Analog input module SM331, $4 \sim 20 \mathrm{~mA}$ current is converted into $0 \sim 27648$ digital quantity by SM331 and send to the CPU.

(2) Temperature detection

The detection of motor temperature and fan shaft temperature is the important parameters in the monitoring system of mine ventilator, When the temperature is too high will lead to the fan doesn't work.Therefore, it is necessary to detect the temperature. The temperature sensor is mainly used to collect the temperature of the front and back bearing temperature, the temperature of the motor stator, the support bearing and so on in this system. Because of the high resistance of the platinum thermal resistance and the measurement accuracy, this system uses PT100 platinum thermal resistance temperature sensor to collect the parameters of the system.

(3) Vibration intensity detection

Detecting the vibration of the fan bearing on the horizontal direction and vertical direction, the system adopts the combination of vibration displacement sensor and transmitter, installed at the front and rear bearing of motor vibration sensor, the vibration intensity on the motor bearing (in $\mathrm{mm} / \mathrm{s}$ ) linear is converted into $4 \sim 20 \mathrm{~mA}$ current signal to module SM331, and convert current signal to digital quantity between 0 and 27, 648 by the module SM331 and sent to CPU.

(4) Measurement of electrical parameters

Electric parameter is an important index to evaluate the performance of fan, the monitoring of electrical parameters can understand the current running state of the fan and the fault state of the motor, which is conducive to safe operation. The electrical parameters of the system include three phase voltage, three-phase current, active power and reactive power, power frequency, power factor and other parameters. The system uses the power parameter transmitter to monitor the electrical parameters, and the RS485 bus mode is adopted to transmit power parameters.

(5) Measurement of gas concentration

The main component of the gas is methane. The measurement of gas concentration is selected at the air outlet of the fan or at the bottom of the mine. In order to control the concentration accurately, this system chooses the mine methane sensor in the air outlet and the bottom at the same time to measure the concentration of methane, the sensor has the acousto-optic alarm and display, With multiple signal output formats and alarm ,display function, can be matched with the signal acquisition and monitoring system of coal mine.

\subsection{Frequency conversion equipment}

The frequency conversion system for one driving two, a frequency converter control two main fan running at the same time. System control the running speed of the fan, improve the operation of industrial and mining, saving energy through the inverter. This system uses the wind turbine dedicated MM430 converter, MM430 built-in PID controller, with high flexibility and reliability,With perfect 
inverter over voltage, over temperature, short circuit protection and other functions, The output power range from $7.5 \mathrm{KWto} 250 \mathrm{KW}$, which has an integrated 485 communication interface, and the frequency conversion device comprises an automatic bypass cabinet.

5. The software design

The software design of the system mainly has two parts: the software design of the total control center PLC and the software design of the upper computer, two parts control the whole system.

5.1 software design of the control system

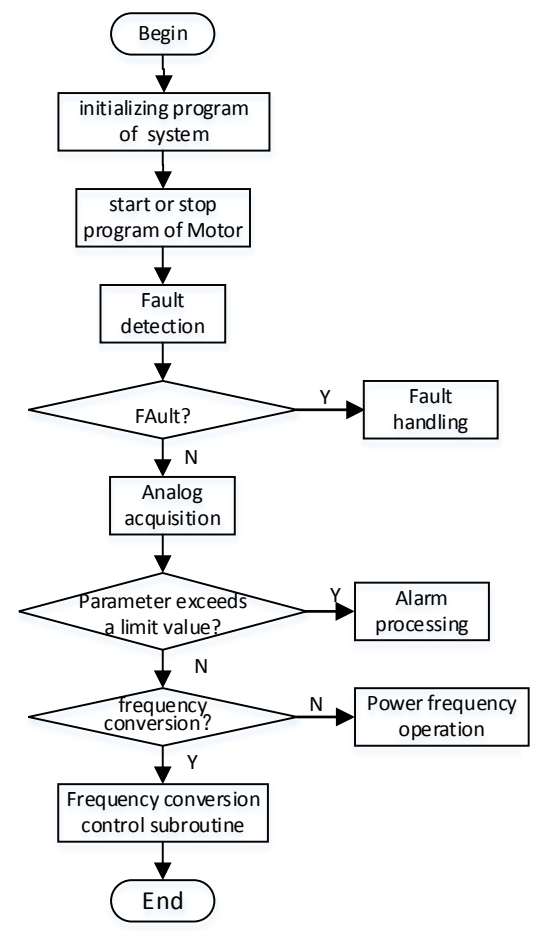

Fig. 3.The main program flow chart

This system uses STEP-7 programming. The PLC program section includes the main program and the control program. Among them, the main program includes system initialization program, motor start, stop control program, fault diagnosis and alarm, frequency control program, analog acquisition process and other functions. The initialization part includes Initialization of various preset parameter values, detection hardware system and power supply, After the confirmation process accurately, start the control system, Analog acquisition program has collected gas concentration, temperature, air pressure, vibration signal and the conversion function. The main program flow chart as shown in figure 3.

\subsection{Monitoring software of upper computer}

The PC monitor software of this system USES Siemens WinCC configuration softwar. Configuration software is the interactive interface between the fan and the staff. It is the core of the whole system to monitor, control, schedule and manage the whole system. Monitor screen can display the monitoring parameter information, alarm and equipment fault conditions, the operator to determine the scene of the wind turbine work status and fault information, etc. PC monitoring system of the main interface as shown in figure 4. 


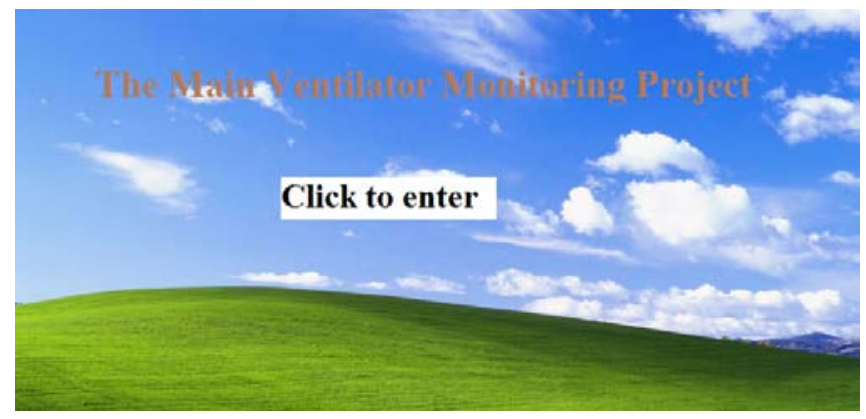

Fig. 4 Main frame of monitoring system

Fan screen is showed in figure 5. In the fan screen,it can monitor and operation related parameters of the fan frequency converter,Including frequency, current, fan switch state ,each contactor switch state of Frequency conversion switch cabinet, it can operate KM3、KM4、KM5 in the pc.

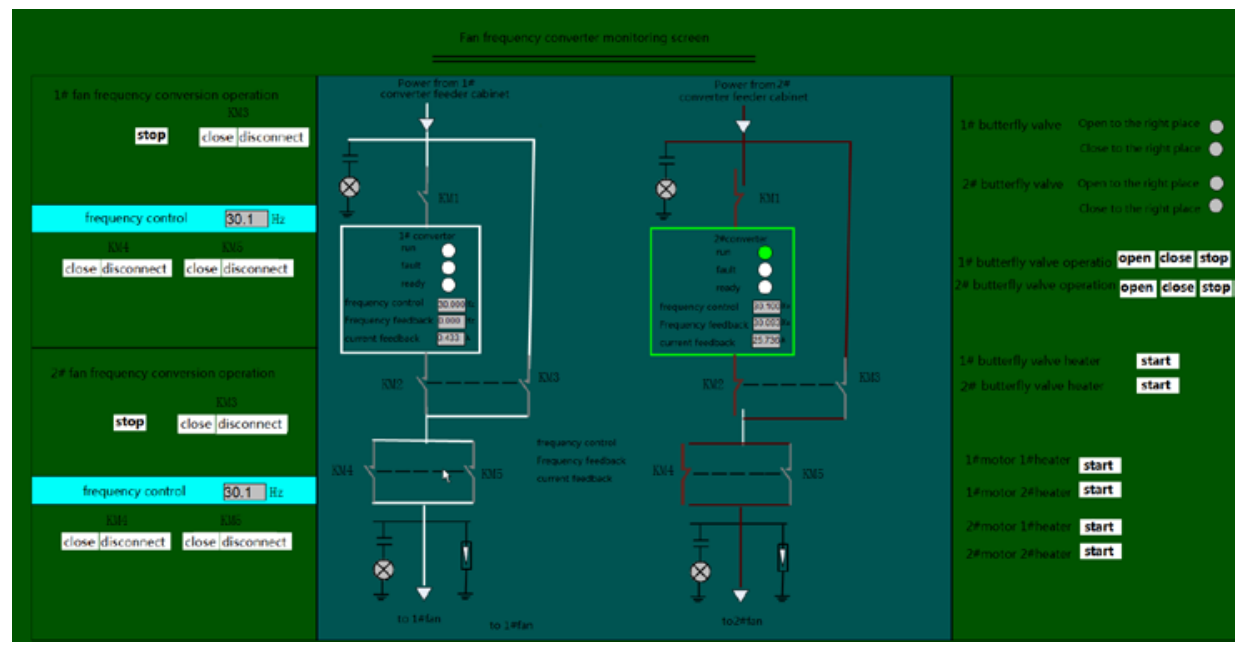

Fig. 5 Fan screen

\section{Conclusion}

On line monitoring system of mine frequency conversion fan based on PLC. The system has realized the monitoring of fan operation status, and has realized the detection and diagnosis of fan fault. It is very good to ensure the safety of coal mine production. The system has good interactivity, can query, display and control system on the same platform, The system had good results in the use of the process.

\section{Acknowledgements}

This paper is fund Project supported by key scientific research projects of Henan provincial colleges (15B413008)

\section{References:}

[1]Ma wen-hui,Fan yong-tai,Chen Hui.Development of coal mine ventilator real-time monitoringSystem based on PLC S7-300[J].CoalTechnology.2013(02):23-24

[2]HAN Tao,Huang You-rui,Qu li-guo.Wireless monitoring system of ventilators based on zigbee in mine[J].CoalMineMachinery.2014(01):218-219

[3]Nie dan-feng.Reformand design of control system for local ventilator[D].Chengdu university of technology.2009:22-24 
[4]Wang yue-wei.Study on coal mine fan monitoring system based on OPC and PLC[J].CoalMineMachinery.2013(10):212-214

[5]Lin Lin,Wang ding-yuan,Xu fei.Research on Intelligent Manager administer and remote monitor system of mine ventilators[J].safety in coal mines.2008(02):22-23

[6] Orkisz, M.Variable frequency drive as a source of condition monitoring data,Institute of Electrical and Electronics Engineers Computer Society, Institute of Electrical and Electronics Engineers Computer Society Jun 11-13 2008. 